\title{
Preparations and Properties of Polyurethane Adhesives Modified by Corn Straw Lignin
}

\author{
Nan Sun, ${ }^{a}$ Yayun Lai, ${ }^{\text {a,b }}$ Yushu Xu, ${ }^{\mathrm{a}}$ Luoqing Wang, ${ }^{\mathrm{a}}$ Xinyu Shang, ${ }^{\mathrm{a}}$ Mingwei Di, ${ }^{\mathrm{a}, *}$ and \\ Xianzhi Kong ${ }^{c}, *$
}

The conversion of lignin into valuable products has attracted the interest of researchers. A series of modified polyurethane adhesives were prepared by blending corn straw enzymatic hydrolyzed lignin with polyester polyol and tolylene-2,4-diisocyanate. Mechanical properties, chemical structures, and thermal stability of the adhesives were characterized by mechanical properties tests, Fourier transform infrared spectrometry (FTIR), and thermogravimetric analysis (TGA). The results of shear strength test under room temperature and high temperature showed that the shear strength for modified polyurethane adhesives was improved by introduction of lignin. The introduction of lignin also improved the heat resistance of polyurethane adhesive. The TGA analysis results showed there were two stages in the thermal decomposition of the lignin blend modified polyurethane adhesive, and the maximum decomposition temperature of the first stage increased with the increase of lignin content, while the maximum decomposition temperature of the second stage decreased with the increase of lignin content. The TGA-FTIR combination analysis studied the main gas generated in the two decomposition stage's peak times, of which $\mathrm{CO}_{2}$ was produced in the first stage, and $\mathrm{CH}_{4}$ was created in the second stage, indicating that the molecular chain fracture process of the two kinds of adhesives was similar in the whole decomposition process.

Keywords: Polyurethane adhesive; Blending modification; Corn straw enzymatic hydrolyzed lignin; Bonding properties; Heat resistance

Contact information: a: Key Laboratory of Bio-based Material Science and Technology (Ministry of Education), Northeast Forestry University, Harbin, China; b: Afforestation Committee of Taiyuan,

Taiyuan, China; c: Institute of Petrochemistry, Heilongjiang Academy of Sciences, Harbin, China;

*Corresponding authors: dimingwei@126.com; kongxianzhi@hotmail.com

\section{INTRODUCTION}

As one of the most abundant organic biopolymers on earth, lignin is the only nonpetroleum resource in nature that can provide renewable aryl compounds. However, at present, the use of lignin is not complete. Because of its complex chemical structure and difficult separation and extraction, lignin is often burned as a cheap solid fuel to recover part of the heat energy, and only a small part of it is used in high value-added products (Markarian 2005). Therefore, improving the use value of lignin and expanding the scope of use not only can improve the utilization rate of resources, but also it can contribute to the protection of the environment, helping to meet the requirements of today's green and environmental protection era. Lignin, with a three-dimensional amorphous structure, is a polyphenol natural macromolecular organic matter formed by the connection of a C-C bond and -O- bond with phenylpropane structural unit (Collins et al. 2019). The main chemical functional groups in the lignin molecule's structure include the aromatic, 
hydroxyl, methoxyl, carbonyl, and carboxyl groups, etc. (Yang et al. 2007; Ralph et al. 2019). Its microstructures make it an interesting raw material for a wide variety of applications. Due to its phenolic ether structure, lignin can be used in blends with other polymers to improve the heat-resistance of the polymers (Zakzeski et al. 2010).

Polyurethane adhesive is a type of reactive adhesive with excellent properties. The isocyanate group and urethane group contained in the polyurethane molecular chain segment are strong in polarity, good chemical activity, and excellent chemical adhesion toward materials containing active hydrogen components (Desai et al. 2003; Tavares et al. 2016; Tenorio-Alfonso et al. 2019). In addition, polyurethane adhesive also has a high degree of flexibility in formulation design, reaction activity, excellent impact resistance, and chemical resistance, which introduces a wide variety of applications of the polyurethane (Osman et al. 2003). However, at present, the polyurethane raw materials produced are all derived from petrochemical derivatives. This kind polyurethane is not easy to degrade, and this can lead to problems, such as soil deterioration and air pollution, in the treatment of its waste. Hence, renewable resources are more advantageous used as precursors to produce polyurethane and provide a more sustainable route for the production of polyurethane. The methoxy, hydroxyl and ether bonds in the lignin structure make it a potential substitute for polyols in several polyurethane materials, such as resins.

In the past, researchers studied lignin-based polyurethane products, such as thermoplastics, elastomers, coatings, film adhesives, etc. (Saito et al. 2013; Chauhan et al. 2014; Jia et al. 2015; Lee and Deng 2015; Oluwasina et al. 2015; Zhang et al. 2015; Sanjiv Kasbe et al. 2017; Gadhave et al. 2018). However, the chemical modification of lignin before preparation polyurethane increased the cost of preparation, while at the same time, the complex chemical and macromolecular structure of lignin made the elasticity and brittleness control of products difficult. These may be some of the reasons that limited its application in lignin-based polyurethane adhesives. The lignin used in this study has not been treated by complex chemical treatment and the lignin-based polyurethane adhesive was prepared by simple blending, which reduced the complexity of the experimental operation and reduces the experimental cost. In this work, a series of bi-component blended polyurethane adhesives were obtained by the unchemically modified corn straw enzymatic hydrolyzed lignin mixed with polyester polyol and tolylene-2,4-diisocyanate (TDI). The effect of different lignin additions on the bonding strength under room temperature and high temperature were studied. At the same time, it lays a foundation for the application of lignin in other adhesives.

\section{EXPERIMENTAL}

\section{Materials}

Corn straw enzymatic hydrolyzed lignin was obtained from Songyuan Laihe Chemicals (Jilin, China), with a bulk density of $0.6 \mathrm{~g} / \mathrm{cm}^{3}$, an average particle size of 11 $\mu \mathrm{m}$ and the purity of $90 \%$. The lignin was used after drying at $50{ }^{\circ} \mathrm{C}$ for $24 \mathrm{~h}$ in a vacuum oven. The phenolic hydroxylcontent of the lignin was $3.64 \%$, and the alcohol hydroxyl content of lignin was $7.62 \%$ (hydroxyl content data is provided by the manufacturer). Polyester polyol $\left(M_{\mathrm{n}}=2000\right.$, acid value $=12.9 \mathrm{mgKOH} / \mathrm{g}$, hydroxyl value $=160.4$ $\mathrm{mgKOH} / \mathrm{g}$ ), which was prepared from sebacic acid, ethylene glycol, and glycerol, was provided by Petroleum Chemistry Research Institute of Heilongjiang Academy of Sciences (Harbin, China). Tolylene-2,4-diisocyanate (TDI) (chemically pure, Wanhua Chemical 
Group Co., Ltd., Yantai, China), dibutyltin dilaurate (DBTDL) (chemically pure, Sinopharm Chemical Reagent Co., Ltd., Shanghai, China), triethylenediamine (TEDA) (analytically pure, Sinopharm Chemical Reagent Co., Ltd., Shanghai, China), acetone, butanone (analytically pure, Sinopharm Chemical Reagent Co., Ltd., Shanghai, China) were used as received. The substrate material used in the adhesive joints was aluminum alloy.

\section{Preparation of Adhesive and Adhesion of Substrate Material}

The lignin was added to a polyester polyol, heated to a molten state at $100{ }^{\circ} \mathrm{C}$, and mixed for $30 \mathrm{~min}$. The catalyst, DBTDL and TEDA, were added into the above mixture and stirred for another $30 \mathrm{~min}$ to prepare the A component of the polyurethane adhesive. The TDI was used as the B component. The polyurethane adhesive blended with lignin was prepared by dissolving A component with acetone, then adding B component, and after that it was stirred at room temperature every $20 \mathrm{~min}$ to $30 \mathrm{~min}$ until a paste was formed. A series of lignin blended polyurethane adhesives were prepared with different designed polyester polyol/lignin mass ratios, and the corresponding numbers are listed in Table 1 . The surface of aluminum substrate was mechanically polished and then wiped with acetone before being adhered.

Table 1. Formula of the Two-component Polyurethane Adhesives

\begin{tabular}{|c|c|c|c|c|c|c|}
\hline & Polyester Polyol $(\mathrm{g})$ & Lignin $(\mathrm{g})$ & DBTDL $(\mathrm{g})$ & TEDA $(\mathrm{g})$ & Acetone $(\mathrm{g})$ & TDI $(\mathrm{g})$ \\
\hline BLN-0 & 100 & 0 & 4 & 2 & 100 & 100 \\
\hline BLN-5 & 100 & 5 & 4 & 2 & 100 & 100 \\
\hline BLN-10 & 100 & 10 & 4 & 2 & 100 & 100 \\
\hline BLN-15 & 100 & 15 & 4 & 2 & 100 & 100 \\
\hline BLN-20 & 100 & 20 & 4 & 2 & 100 & 100 \\
\hline
\end{tabular}

\section{Methods}

Single lap shear joints of aluminum substrates with the size of $100 \mathrm{~mm} \times 25 \mathrm{~mm} \times$ $2 \mathrm{~mm}$ were used for evaluating the adhesive shear strength by tension loading with an overlap length of $12.5 \mathrm{~mm}$. The adhesives were applied across both sides of the overlap area and cured at $110^{\circ} \mathrm{C}$ for $3 \mathrm{~h}$. During the curing, the specimens were under a constant pressure of $0.1 \mathrm{MPa}$.

After curing, the tests of the mechanical properties for the polyurethane adhesives were performed on an Instron 4467 machine (Instron (Shanghai) Test Equipment Trading Co., Ltd., Shanghai, China) at the speed of $10 \mathrm{~mm} / \mathrm{min}$ at the room temperature and high temperature, respectively, according to ASTM D-1002 (2010).

Absorbance peaks of the characteristic groups of reaction mixture were obtained with a Fourier transform infrared (FT-IR) instrument (NICOLET-6700; Thermo Electron Corporation, New York, NY, USA).

Thermal properties of the adhesives were performed on the thermal gravimetric analyzer (TGA, NETZSCH TG-209F3; NETZSCH Scientific Instruments Trading (Shanghai) Ltd., Shanghai, China) at a heating rate of $10 \mathrm{~K} / \mathrm{min}$ from 303.15 to $873.15 \mathrm{~K}$ under $\mathrm{N}_{2}$ atmosphere $(30 \mathrm{~mL} / \mathrm{min})$.

The TG-FTIR analysis was obtained using the combination of the TGA Q5000 (TA Instruments, New Castle, DE, USA) and Nicolet 6700 at heating rate of $10 \mathrm{~K} / \mathrm{min}$, and 20 $\mathrm{mL} / \mathrm{min}$ of nitrogen. 


\section{RESULTS AND DISCUSSIONS}

\section{Bonding Properties}

\section{Shear strength under room temperature}

Shear strength test was used to evaluate the bonding properties of the adhesive for the same material, and a single lap structure was used for shear specimens. The effect of lignin content on shear strength of polyurethane adhesives is shown in Fig. 1. In theory, active hydrogen in lignin will react with isocyanate, which affects the strength of polyurethane adhesives. Therefore, the addition of lignin will also affect the shear strength of polyurethane adhesives.

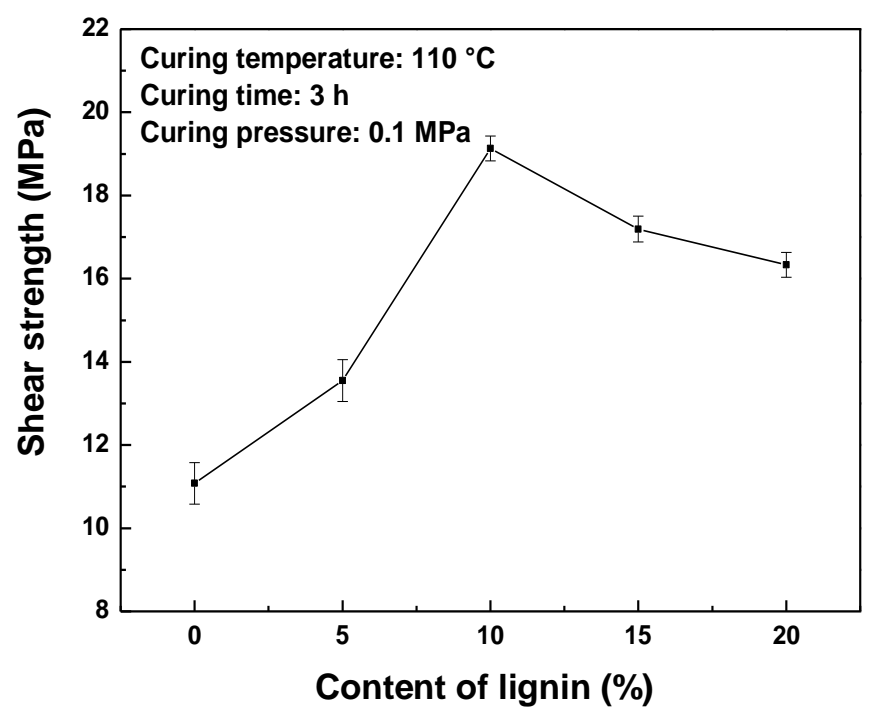

Fig. 1. Room temperature shear strength of the polyurethane adhesives modified by lignin

It can be seen from Fig. 1 that the shear strength of polyurethane adhesive increased at first and then decreased with the addition of lignin. The highest shear strength of polyurethane adhesive was $19.1 \mathrm{MPa}$, which was observed for $10 \%$ lignin addition. When lignin content reached 20\%, the shear strength of polyurethane adhesive was still $16.3 \mathrm{MPa}$. When the lignin content was increased from $10 \%$ to $20 \%$, polyurethane adhesives had a high shear strength, even if the lignin content was 5\%, the shear strength of polyurethane adhesives was 13.6 MPa, which showed that even if the lignin performance had a large fluctuation, the lignin blend modified polyurethane adhesives still had a satisfactory shear strength. When the proportion of lignin was small, the active hydrogen in lignin reacted with isocyanate increases the cross-linking degree and the hydrogen bonding interaction was enhanced with the introduction of lignin, so the shear strength increased. When a certain amount was reached, the three-dimensional structure of lignin destroyed the regularity of molecular chains in the polymer structure of polyurethane adhesives inversely, which reduced the shear strength. The obvious "fault-tolerant" ability of lignin blend modified polyurethane adhesive, which resulted from the corn straw enzymatic hydrolyzed lignin that was insoluble and had good compatibility with polyurethane adhesives, was an important basis for the practical application of lignin in polyurethane adhesive. 


\section{Shear strength under high temperature}

To study the heat resistance of polyurethane adhesive blended with lignin, the shear strength of the bonding joint under various temperatures was also tested. The shear strengths for five polyurethane adhesives BLN-0, BLN-5, BLN-10, BLN-15, and BLN-20 under various temperatures are shown in Fig. 2. When the shear strength tests were performed at high temperatures of $60^{\circ} \mathrm{C}$ and $80^{\circ} \mathrm{C}, \mathrm{BLN}-10$ polyurethane adhesive showed the best bonding property, and BLN-5 showed a bonding property slightly worse than BLN-10. In contrast, when the temperature was $100{ }^{\circ} \mathrm{C}$ and $120^{\circ} \mathrm{C}$, the BLN-5 with lignin content of $5 \%$ showed the maximum shear strength while BLN-10 was weaker but still reached for the bonding strength of $7.37 \mathrm{MPa}$ and $6.84 \mathrm{MPa}$, corresponding to $100{ }^{\circ} \mathrm{C}$ and $120{ }^{\circ} \mathrm{C}$, respectively. With the increase of temperature, the amount of active hydrogen reaction between isocyanate and lignin was not increased; however, due to the large threedimensional structure of lignin, the molecular chain in the polymer structure was damaged, and the degree of damage increased, which led to the decrease of the bonding properties of BLN-10 adhesive. Figure 2 also indicates that a higher temperature of the shear strength test resulted in a weaker bonding property of polyurethane adhesive with the same lignin content. This was because the introduction of lignin improved the heat resistance of polyurethane adhesives, and at the same time, the three-dimensional structure of lignin also destroyed the regularity of molecular chains in the polymer structure of polyurethane adhesives. This destruction increased the toughness of polyurethane adhesives, also reduced the crosslinking degree of polyurethane adhesive, resulting in the reduction of high temperature shear strength of polyurethane adhesives.
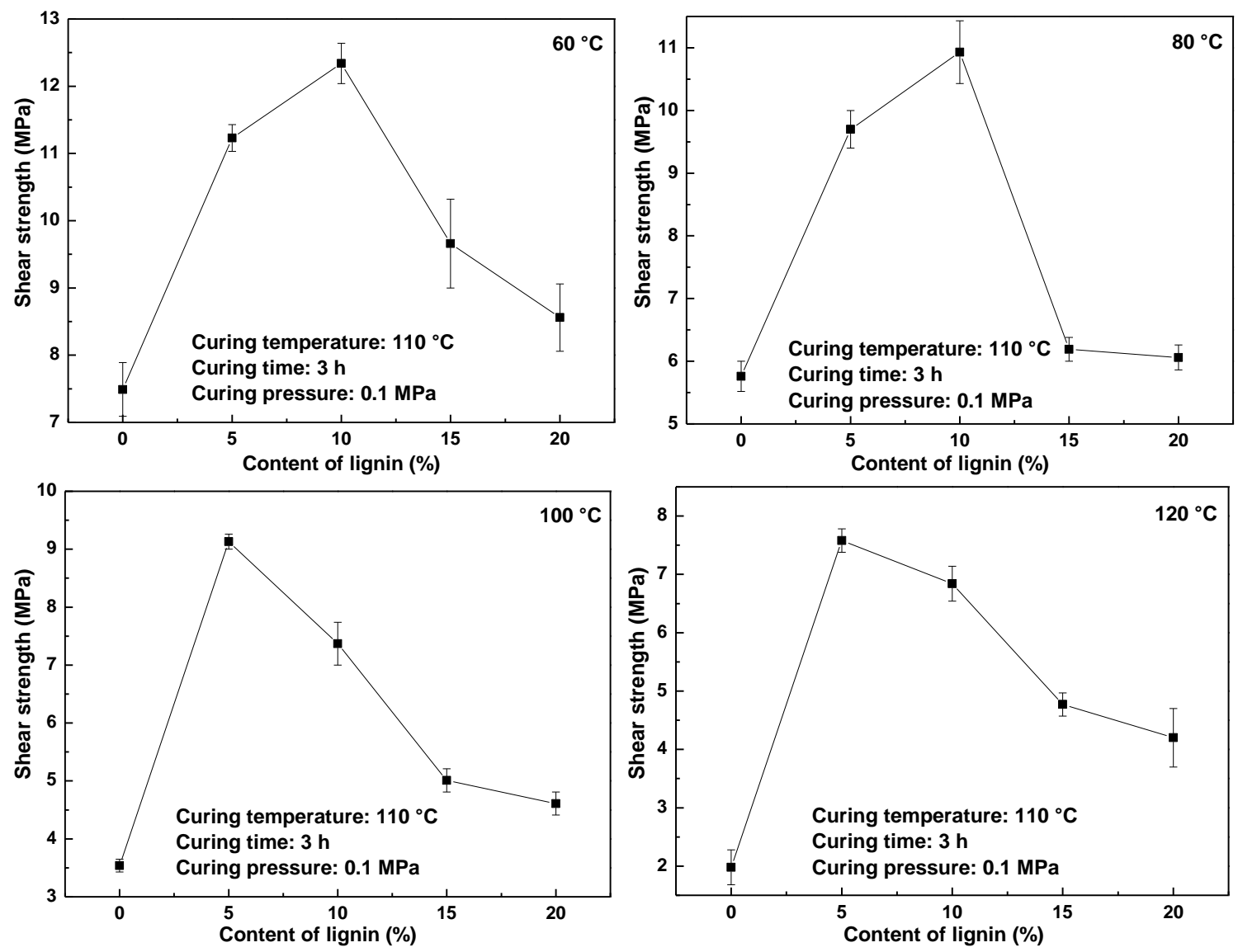

Fig. 2. Effect of lignin content on high temperature shear strength of the polyurethane adhesive 
Figure 3 shows the shear strength of the polyurethane adhesives blended with lignin at different testing temperatures. Table 2 lists the decline rate of high temperature shear strength of lignin blend modified polyurethane adhesives compared with the room temperature shear strength. Although the introduction of lignin increased the strength at room and high temperature, the decrease rate of high temperature shear strength was reinforced with the increase of lignin content under the same temperature, which was due to the decrease of the regularity of polymer chain arrangement in polyurethane adhesive that was caused by the increase of lignin content.
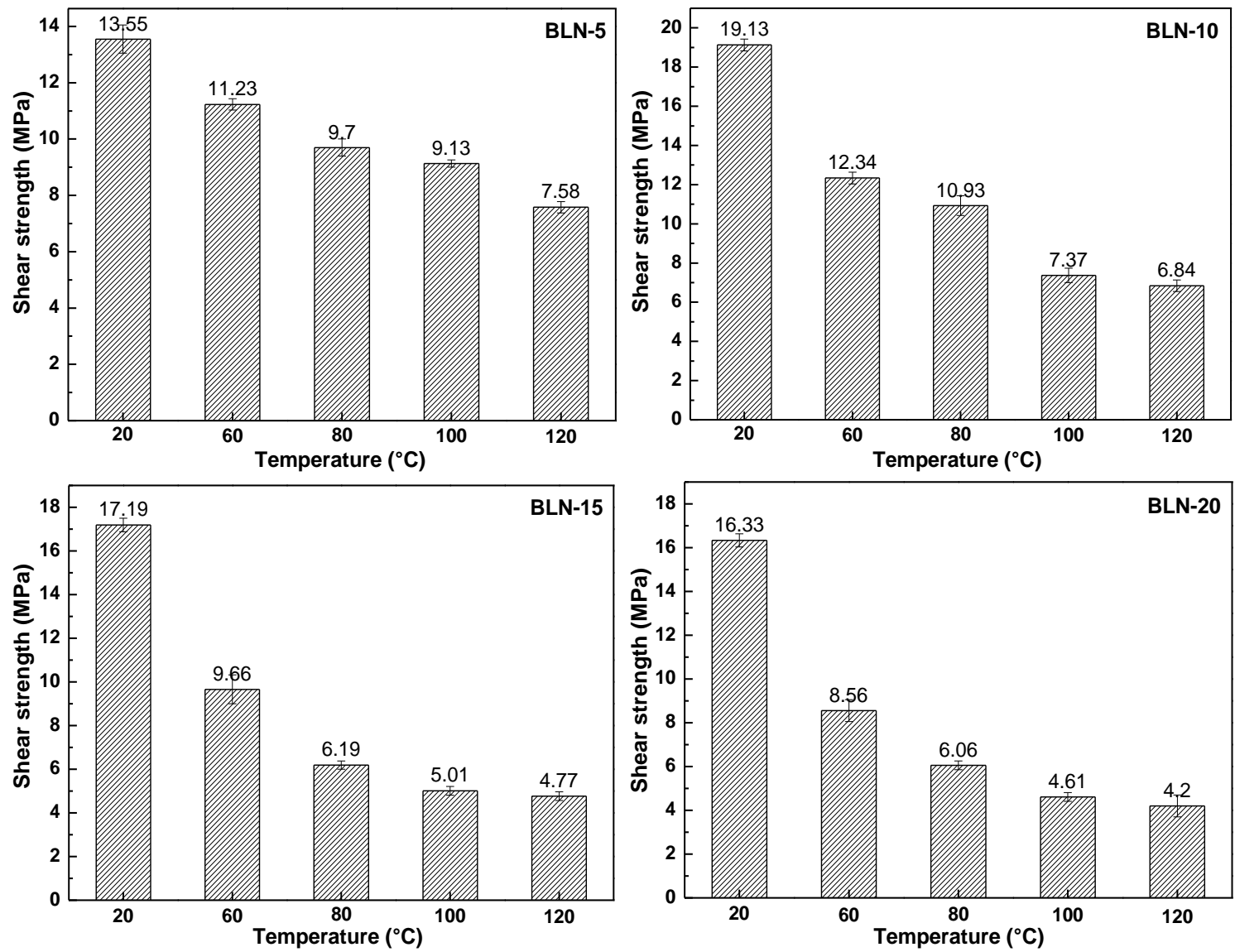

Fig. 3. Shear strength of the polyurethane adhesives blended with lignin at different testing temperature

Table 2. Decline Rate of High-temperature Strength of the Polyurethane Adhesives Blended with Lignin

\begin{tabular}{|c|c|c|c|c|}
\hline $\begin{array}{c}\text { Polyurethane } \\
\text { Adhesive }\end{array}$ & $\begin{array}{c}60^{\circ} \mathrm{C} \\
\text { Decline Rate (\%) }\end{array}$ & $\begin{array}{c}80^{\circ} \mathrm{C} \\
\text { Decline Rate (\%) }\end{array}$ & $\begin{array}{c}100^{\circ} \mathrm{C} \\
\text { Decline Rate } \\
(\%)\end{array}$ & $\begin{array}{c}120^{\circ} \mathrm{C} \\
\text { Decline Rate } \\
(\%)\end{array}$ \\
\hline BLN-5 & 17.1 & 28.4 & 32.6 & 44.1 \\
\hline BLN-10 & 35.4 & 42.9 & 61.5 & 64.2 \\
\hline BLN-15 & 43.8 & 64.0 & 70.9 & 72.3 \\
\hline BLN-20 & 47.6 & 62.9 & 71.8 & 74.3 \\
\hline
\end{tabular}

Ordinary polyurethane adhesives are generally not used as temperature-resistant adhesives, which are determined by the molecular structure of polyurethane materials. In 
polyurethanes synthesized from alcohols and isocyanates, the urethane bonds and maximum decomposition temperature in polyurethane molecular structure are different due to the reaction of different alcohols and isocyanates (Chattopadhyay and Webster 2009; Del Saz-Orozco et al. 2015). The heat resistance of adhesives depends not only on the heat resistance of the molecular structure, but also on the toughness, wettability, and the regularity between molecular chains. Lignin is a natural polyphenolic hydroxyl polymer, and the existence of a large number of polar groups in lignin improves the hydrogen bond interaction and thus improves the heat resistance of polyurethane.

\section{FTIR Analysis}

The FTIR spectra for the polyurethane adhesives with different lignin content cured at $110{ }^{\circ} \mathrm{C}$ for $3 \mathrm{~h}$ are shown in Fig. 4. The FTIR spectrum analysis was employed to characterize the raw materials and analyze the changes in the lignin-polyurethane adhesives structure during its curing reaction, mainly to observe the changes of functional groups introduced by the addition of different amounts of lignin within the adhesive.

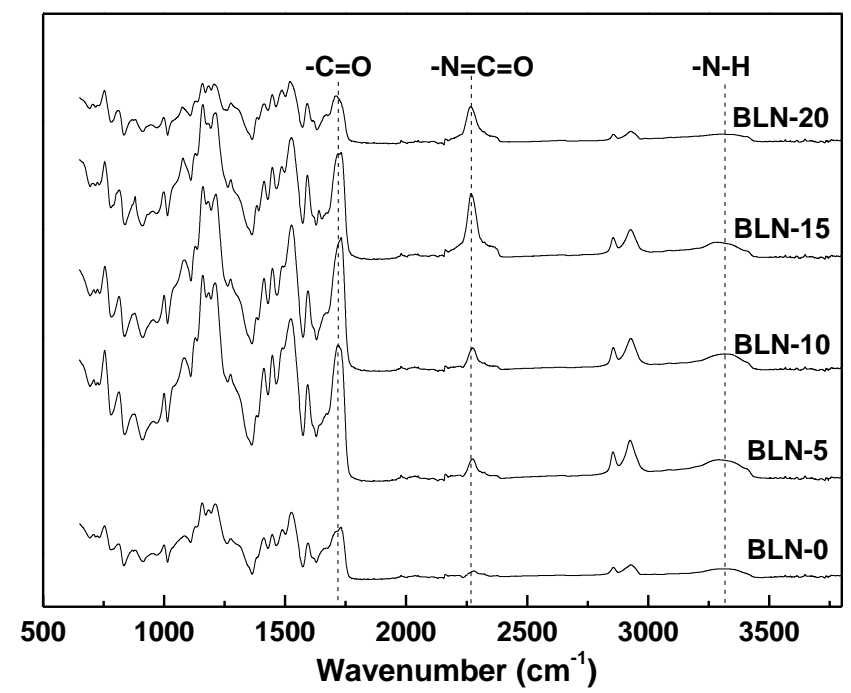

Fig. 4. FTIR spectra of cured polyurethane adhesives blended with lignin

It can be seen from Fig. 4 that the absorbance peak at $2270 \mathrm{~cm}^{-1}$ that was attributed to the stretching vibration of isocyanate group $(\mathrm{N}=\mathrm{C}=\mathrm{O})$ for polyurethane adhesives showed a strengthened peak with the increase of lignin until the lignin additions reached $15 \%$. There were still isocyanate groups after curing of polyurethane adhesives modified by lignin, and the hydrogen bond between lignin and molecular chain increased, and the molecular chain in polyurethane was arranged more closely, which improved the water resistance of polyurethane adhesives and made it an idea of formulation design. The polyurethane adhesives without lignin had almost no stretching vibration peak of isocyanate groups, which indicated that isocyanate and polyester polyol reacted completely. When the lignin content was $5 \%$ and $10 \%$, there was a stretching vibration peak of isocyanate with low peak intensity, indicating that the introduction of lignin influenced the reaction between isocyanate and polyester polyol to some extent. On the one hand, lignin partially hindered the reaction between isocyanate and polyester polyol. On the other hand, lignin can still react with isocyanate, which led to the increase of shear strength. When the lignin content continued to increase, the stretching vibration peak of 
isocyanate intensified, and the three-dimensional network structure of lignin seriously hindered the reaction between isocyanate and hydroxyl group, which resulted in the decrease of shear strength. The result further indicated that the lignin could participate in the crosslinking curing reaction of polyurethane adhesives due to the increase of the multifunctional reactive groups, accompanied by the hydrogen bond between lignin and molecular chain, which resulted in a better performance of the adhesive. However, the addition of lignin interfered with the reaction between isocyanate and hydroxyl because of its three-dimensional network structure. The residual isocyanate groups reacted with water and further crosslinked, which was beneficial to the water resistance of the adhesives.

There were a large number of functional groups that can form a hydrogen bond in polyurethane, such as carbonyl, ether oxygen, and amine. The formation of hydrogen bond changes the bond force constant of the functional group, which is reflected that the stretching vibration band shifts to low frequency, and the intensity and peak shape of the absorbance band also changed in the infrared spectrum (Chattopadhyay and Raju 2007). Among them, the amino group region $\left(3100 \mathrm{~cm}^{-1}\right.$ to $\left.3500 \mathrm{~cm}^{-1}\right)$ and carbonyl group region $\left(1600 \mathrm{~cm}^{-1}\right.$ to $\left.1800 \mathrm{~cm}^{-1}\right)$ are the most interesting spectral regions in the study of hydrogen bonding. The absorbance peak near $1730 \mathrm{~cm}^{-1}$ of the carbamate had the same change trend along the additions of lignin (Cateto et al. 2008; Ignat et al. 2011). With BLN-0 as a reference, the peak intensity greatly increased with $5 \%$ and $10 \%$ lignin addition in the polyurethane adhesives, and then the peak value decreased gradually, even lower than that of BLN-0. The polyurethane adhesives modified with lignin had an absorbance peak near $3310 \mathrm{~cm}^{-1}$, which was due to the stretching vibration of hydroxyl group and $\mathrm{N}-\mathrm{H}$ bonded with ammonia ester (NHCOO). The addition of lignin hindered the reaction of hydroxyl with isocyanate and the existence of hydroxyl in lignin itself, which resulted in the content of hydroxyl increased with the increase of lignin content. The peak values of N-H of BLN5 and BLN-10 were higher, a large number of hydrogen bonds existed, and the strength of the adhesives was stronger. This result was consistent with the analysis on the bonding properties of the adhesives performed at room temperature mentioned above.

\section{TGA Analysis}

Figure 5 shows the TGA and derivative thermogravimetric (DTG) curves of lignin blend-modified polyurethane adhesives. It can be seen that the weight loss of lignin blending modified polyurethane adhesive can be divided into two stages.

The two stage's maximum decomposition temperatures of polyurethane adhesives modified by lignin are shown in Table 3. The maximum decomposition temperature of first stage increased with the increase of lignin content, and the maximum decomposition temperature of BLN-5/BLN-10/BLN-15 lignin modified polyurethane adhesives decreased after the introduction of lignin in the second stage. In the first stage, the intermolecular interaction force was destroyed under high temperature. However, higher lignin content resulted in a stronger intermolecular interaction force, such as hydrogen bonds, and compared with BLN-0, more energy was needed for destruction, which resulted in the increase of thermal decomposition temperature. The second stage was the chemical bond breaking in the molecular chain. The three-dimensional network structure of lignin destroyed the reaction between isocyanate and polyester polyol. Higher lignin content caused weaker chemical bond formation in the molecular chain, which resulted in a thermal decomposition of polyurethane adhesives under lower temperature. 

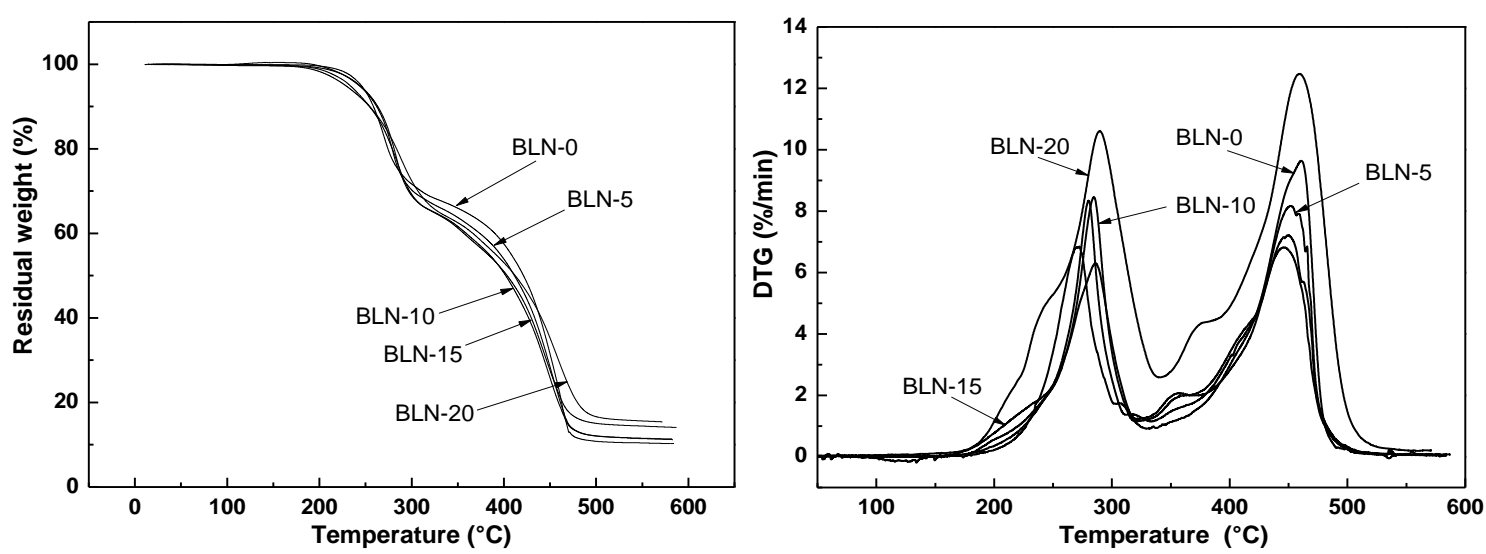

Fig. 5. The TGA and DTG curves of polyurethane adhesives modified by lignin

When lignin was introduced into polyurethane adhesives, the thermogravimetric loss of polyurethane adhesives increased generally before $500{ }^{\circ} \mathrm{C}$. When the temperature reached $500{ }^{\circ} \mathrm{C}$, the weight loss of polyurethane adhesive was lower than that of the pure polyurethane adhesive. This indicated that weak chemical forces, such as hydrogen bond between lignin and molecules, caused weight loss at a low temperature, but the phenol group of lignin at high temperature hindered the degradation of polyurethane adhesives (Ciobanu et al. 2004).

Table 3. Maximum Decomposition Temperature of Polyurethane Adhesives Modified by Lignin

\begin{tabular}{|c|c|c|c|c|c|}
\hline $\begin{array}{c}\text { PU } \\
\text { Adhesives }\end{array}$ & $\mathrm{BLN}-0\left({ }^{\circ} \mathrm{C}\right)$ & $\mathrm{BLN}-5\left({ }^{\circ} \mathrm{C}\right)$ & $\mathrm{BLN}-10\left({ }^{\circ} \mathrm{C}\right)$ & $\mathrm{BLN}-15\left({ }^{\circ} \mathrm{C}\right)$ & $\mathrm{BLN}-20\left({ }^{\circ} \mathrm{C}\right)$ \\
\hline First stage & 271.68 & 279.30 & 283.91 & 287.10 & 291.58 \\
\hline Second stage & 460.90 & 452.26 & 447.54 & 448.59 & 461.63 \\
\hline
\end{tabular}

\section{Crosslinking Degree Analysis}

The crosslinking degree has a considerable effect on the elongation, strength, and medium resistance of polyurethane adhesive. The swelling equilibrium method was used to explore the effect of the introduction of lignin on the crosslinking degree of polyurethane adhesive. Figure 6 shows the crosslinking degree of BLN-0, BLN-1, BLN-2, BLN-3, BLN4, and BLN- 5 adhesives.

It can be seen from Fig. 6 that the introduction of lignin can increase the crosslinking degree of polyurethane adhesive. Among them, the cross-linking degree of BLN-5 was the highest, and it was 30.2\% higher than that of BLN-0. The cross-linking degree of BLN-10 was the lowest, but it was also $13.7 \%$ higher than that of BLN-0. It follows that the introduction of lignin will increase the cross-linking degree of the adhesive to a certain extent. And too much lignin will decrease the cross-linking degree of the material. This is because lignin is a three-dimensional reticular macromolecular structure, which is not conducive to the regular arrangement and distribution of polymer chains in polyurethane adhesives, and also has an adverse effect on the heat and water resistance of polyurethane adhesives. However, this three-dimensional reticular macromolecular structure of lignin can hinder the further damage of heat and water to the polymer chain in the process of heating and water treatment of polyurethane adhesive, thus reducing the fracture number of polymer chain. 


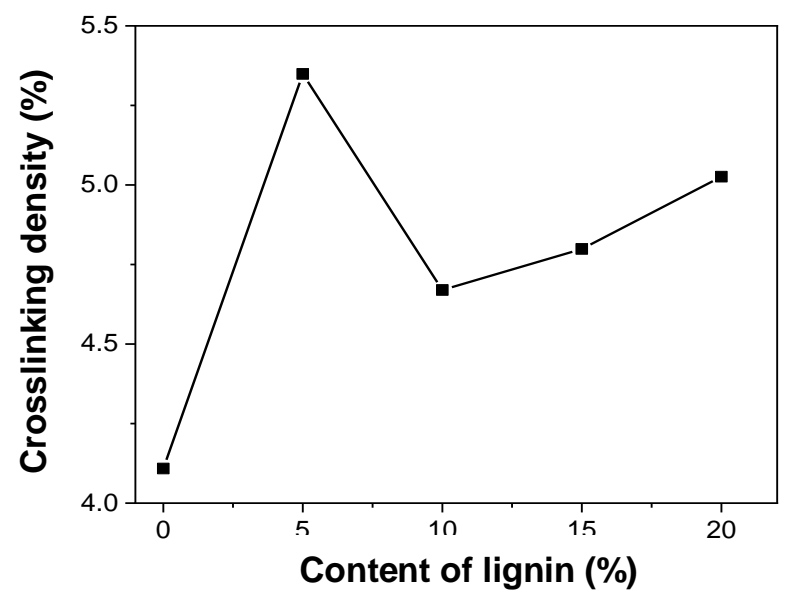

Fig. 6. The effect of lignin content on crosslinking degree of polyurethane adhesives

\section{FTIR-TGA analysis}

To better study the decomposition and functional changes of polyurethane adhesives during thermal degradation, polyurethane adhesives were analyzed by a combination of TGA-FTIR. Figure 7 shows the TGA-DTG curves of BLN-0 and BLN-20 adhesives during TGA-FTIR combination usage stage. The FTIR spectra of the vapor phase for thermal degradation of the two polyurethane adhesives at the peaks of two stages in the decomposition process can be seen from Fig. 8.
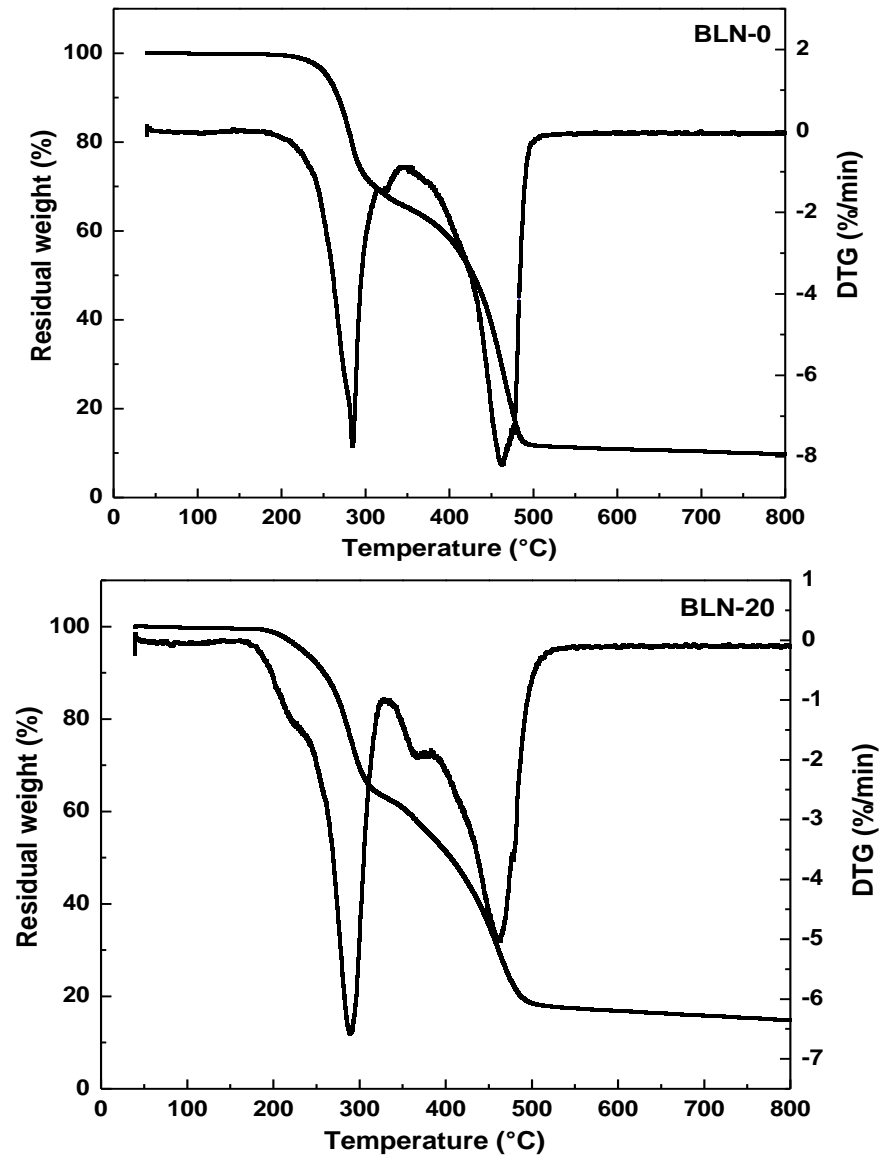

Fig. 7. The TGA-DTG curves of BLN-0 and BLN-20 adhesives 

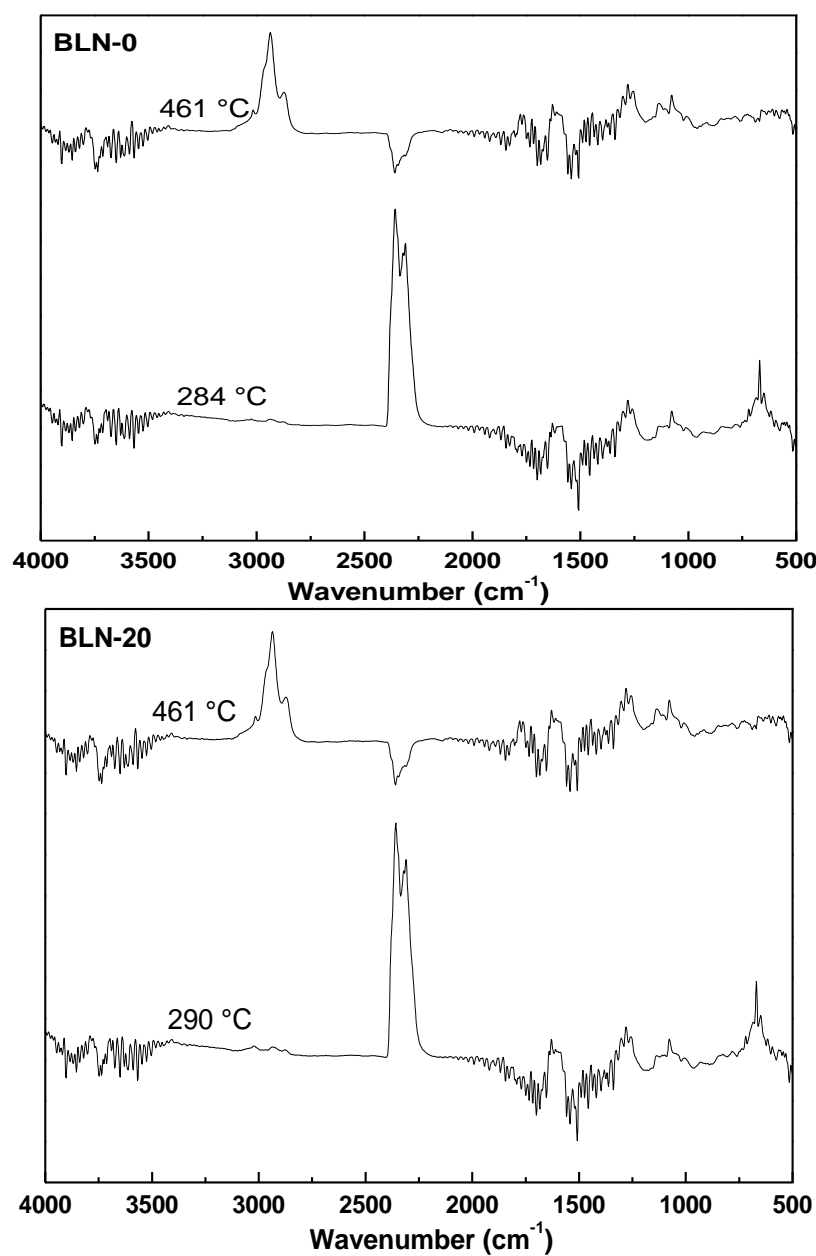

Fig. 8. FTIR spectra of the vapor phases for thermal degradation of polyurethane adhesives BLN0 and $\mathrm{BLN}-20$

Two distinct peaks, C-H $\left(2940 \mathrm{~cm}^{-1}\right)$ and $\mathrm{CO}_{2}\left(2360 \mathrm{~cm}^{-1}\right)$, can be found from Fig. 8. The peaks of $\mathrm{H}_{2} \mathrm{O}$ (4000 to $\left.3500 \mathrm{~cm}^{-1}\right), \mathrm{CO}\left(2120\right.$ to $\left.2180 \mathrm{~cm}^{-1}\right)$, and some phenols and phenolic peaks still were present, but were not obvious. According to Lambert-Beer's law, the gas product concentration is proportional to the absorbance intensity of the corresponding infrared spectrum. A larger infrared spectrum absorbance intensity value results in a higher gas concentration generated by pyrolysis. Therefore, the main gas generated at the first stage peak was $\mathrm{CO}_{2}$, and the gas produced at the peak of the second stage peak was mainly $\mathrm{CH}_{4}$. Figure 8 also shows that there was no obvious difference between the peak values of the two phases, which indicated that the molecular chain fracture process of the two kinds of adhesives was similar in the whole decomposition process. This may be due to the fact that in the first stage, the carbamate group on the polymer backbone breaks down at the $\mathrm{C}-\mathrm{O}$ bond to form isocyanates and polyols, which are then further decomposed into $\mathrm{CO}_{2}$. In the second stage, when the temperature increases, the broken molecular segment is further decomposed to $\mathrm{CH}_{4}$ (Shufen et al. 2006). 


\section{CONCLUSIONS}

1. A series of modified polyurethane adhesives were prepared by blending corn straw enzymatic hydrolyzed lignin into polyester polyol, and this was reacted with tolylene2,4-diisocyanate (TDI) under catalyst. The strength of modified polyurethane adhesives can be improved by introduction of lignin in the series of modified polyurethane adhesives. The highest shear strength at room temperature for polyurethane adhesives was found in adhesive BLN-10, which could reach 19.1 MPa. The high temperature strength measurement of polyurethane series adhesives modified by lignin blend showed lower values than that at room temperature, and BLN-5 adhesive had the lowest decrease.

2. The isocyanate group peak for polyurethane adhesives displayed stronger peak intensity with the increase of lignin content until the lignin additions reached 15\%. Addition of lignin not only increased the number of reactive functional groups, but it also hindered the reaction between isocyanate and hydroxyl because of its threedimensional network structure. The thermal decomposition process of lignin blend modified polyurethane adhesives was divided into two stages. The maximum decomposition temperature of the first stage increased with the increase of lignin content, while the decomposition temperature decreased in the second stage.

3. The results of TGA-FTIR analysis of two polyurethane adhesives, BLN-0 and BLN20 , showed that the main gas generated at the first stage peak time was $\mathrm{CO}_{2}$, and the gas produced at the peak of the second stage peak was mainly $\mathrm{CH}_{4}$. The trends of molecular chain fracture process of the two kinds of adhesives were similar in the whole decomposition process.

\section{ACKNOWLEDGMENTS}

This work was supported by the National Natural Science Foundation of China (31670567), the Fundamental Research Funds for the Central Universities of China (2572018CP01), and the Innovation Training Program for College Students of Northeast Forestry University of China (CL201909).

\section{REFERENCES CITED}

ASTM D-1002 (2010). "Standard test method for apparent shear strength of single-lapjoint adhesively bonded metal specimens by tension loading (metal-to-metal)," ASTM International, West Conshohocken, PA, USA.

Cateto, C. A., Barreiro, M. F., and Rodrigues, A. E. (2008). "Monitoring of lignin-based polyurethane synthesis by FTIR-ATR," Industrial Crops and Products 27(2), 168174. DOI: 10.1016/j.indcrop.2007.07.018

Chattopadhyay, D. K., and Raju, K. V. S. N. (2007). "Structural engineering of polyurethane coatings for high performance applications," Progress in Polymer Science (Oxford) 32(3), 352-418. DOI: 10.1016/j.progpolymsci.2006.05.003 
Chattopadhyay, D. K., and Webster, D. C. (2009). "Thermal stability and flame retardancy of polyurethanes," Progress in Polymer Science (Oxford) 34(10), 10681133. DOI: 10.1016/j.progpolymsci.2009.06.002

Chauhan, M., Gupta, M., Singh, B., Singh, A. K., and Gupta, V. K. (2014). "Effect of functionalized lignin on the properties of lignin-isocyanate prepolymer blends and composites," European Polymer Journal 52(1), 32-43. DOI:

10.1016/j.eurpolymj.2013.12.016

Ciobanu, C., Ungureanu, M., Ignat, L., Ungureanu, D., and Popa, V. I. (2004). "Properties of lignin-polyurethane films prepared by casting method," Industrial Crops and Products 20(2), 231-241. DOI: 10.1016/j.indcrop.2004.04.024

Collins, M. N., Nechifor, M., Tanasă, F., Zănoagă, M., McLoughlin, A., Stróżyk, M. A., Culebras, M., and Teacă, C. A. (2019). "Valorization of lignin in polymer and composite systems for advanced engineering applications - A review," International Journal of Biological Macromolecules 131, 828-849. DOI:

10.1016/j.ijbiomac.2019.03.069

Del Saz-Orozco, B., Alonso, M. V., Oliet, M., Domínguez, J. C., Rojo, E., and Rodriguez, F. (2015). "Lignin particle- and wood flour-reinforced phenolic foams: Friability, thermal stability and effect of hygrothermal aging on mechanical properties and morphology," Composites Part B: Engineering 80, 154-161. DOI:

10.1016/j.compositesb.2015.05.043

Desai, S. D., Patel, J. V., and Sinha, V. K. (2003). "Polyurethane adhesive system from biomaterial-based polyol for bonding wood," International Journal of Adhesion and Adhesives 23(5), 393-399. DOI: 10.1016/S0143-7496(03)00070-8

Gadhave, R. V., Mahanwar, P. A., and Gadekar, P. T. (2018). "Lignin-polyurethane based biodegradable foam," Open Journal of Polymer Chemistry 08(1), 1-10. DOI: 10.4236/ojpchem.2018.81001

Ignat, L., Ignat, M., Ciobanu, C., Doroftei, F., and Popa, V. I. (2011). "Effects of flax lignin addition on enzymatic oxidation of poly(ethylene adipate) urethanes," Industrial Crops and Products 34(1), 1017-1028. DOI: 10.1016/j.indcrop.2011.03.010

Jia, Z., Lu, C., Zhou, P., and Wang, L. (2015). "Preparation and characterization of high boiling solvent lignin-based polyurethane film with lignin as the only hydroxyl group provider," RSC Advances 5(66), 53949-53955. DOI: 10.1039/c5ra09477a

Lee, A., and Deng, Y. (2015). "Green polyurethane from lignin and soybean oil through non-isocyanate reactions," European Polymer Journal 63, 67-73. DOI: 10.1016/j.eurpolymj.2014.11.023

Markarian, J. (2005). "Wood-plastic composites: Current trends in materials and processing," Plastics, Additives and Compounding 7(5), 20-26. DOI: 10.1016/S1464391X(05)70453-0

Oluwasina, O. O., Lajide, L., and Owolabi, B. J. (2015). "Performance of bonded boards using lignin-based resins," Wood Material Science and Engineering 10(2), 168-177. DOI: $10.1080 / 17480272.2014 .923044$

Osman, M. A., Mittal, V., Morbidelli, M., and Suter, U. W. (2003). "Polyurethane adhesive nanocomposites as gas permeation barrier," Macromolecules 36(26), 98519858. DOI: $10.1021 / \mathrm{ma035077x}$

Ralph, J., Lapierre, C., and Boerjan, W. (2019). "Lignin structure and its engineering," Current Opinion in Biotechnology 56, 240-249. DOI: 10.1016/j.copbio.2019.02.019 
Saito, T., Perkins, J. H., Jackson, D. C., Trammel, N. E., Hunt, M. A., and Naskar, A. K. (2013). "Development of lignin-based polyurethane thermoplastics," RSC Advances 3(44), 21832-21840. DOI: 10.1039/c3ra44794d

Sanjiv Kasbe, P., Kumar, N., and Manik, G. (2017). “A molecular simulation analysis of influence of lignosulphonate addition on properties of modified 2-ethyl hexyl acrylate/methyl methacrylate/acrylic acid based pressure sensitive adhesive," International Journal of Adhesion and Adhesives 78, 45-54. DOI: 10.1016/j.ijadhadh.2017.06.014

Tavares, L. B., Boas, C. V., Schleder, G. R., Nacas, A. M., Rosa, D. S., and Santos, D. J. (2016). "Bio-based polyurethane prepared from Kraft lignin and modified castor oil," Express Polymer Letters 10(11), 927-940. DOI: 10.3144/expresspolymlett.2016.86

Tenorio-Alfonso, A., Sánchez, M. C., and Franco, J. M. (2019). "Synthesis and mechanical properties of bio-sourced polyurethane adhesives obtained from castor oil and MDI-modified cellulose acetate: Influence of cellulose acetate modification," International Journal of Adhesion and Adhesives 95, Article Number 102404. DOI: 10.1016/j.ijadhadh.2019.102404

Yang, H., Yan, R., Chen, H., Lee, D. H., and Zheng, C. (2007). "Characteristics of hemicellulose, cellulose and lignin pyrolysis," Fuel 86(12-13), 1781-1788. DOI: 10.1016/j.fuel.2006.12.013

Zakzeski, J., Bruijnincx, P. C. A., Jongerius, A. L., and Weckhuysen, B. M. (2010). "The catalytic valorization of lignin for the production of renewable chemicals," Chemical Reviews 110(6), 3552-3599. DOI: 10.1021/cr900354u

Zhang, C., Wu, H., and Kessler, M. R. K. (2015). "High bio-content polyurethane composites with urethane modified lignin as filler," Polymer 69(1), 52-57. DOI: 10.1016/j.polymer.2015.05.046

Shufen, L., Zhi, J., Kaijun, Y., Shuqin, Y., and Chow, W. K. (2006). "Studies on the thermal behavior of polyurethanes," Polymer-Plastics Technology and Engineering 45(1), 95-108. DOI: 10.1080/03602550500373634

Article submitted: January 8, 2020; Peer-review completed: March 21, 2020; Revised version received and accepted: April 5, 2020; Published: April 8, 2020.

DOI: 10.15376/biores.15.2.3970-3983 